\title{
Association of sugar-sweetened beverage intake at 18 months and 5 years of age with adiposity outcomes at 6 years of age: the Singapore GUSTO mother-offspring cohort
}

Phaik Ling Quah ${ }^{1}$, Josefien Kleijweg ${ }^{1}$, Ya Yin Chang ${ }^{1}$, Jia Ying Toh ${ }^{1}$, Hui Xian Lim ${ }^{1}$, Ray Sugianto ${ }^{2}$, Izzuddin M. Aris ${ }^{1,3}$, Wen Lun Yuan ${ }^{4}$, Mya Thway Tint ${ }^{3}$, Jonathan Y. Bernard ${ }^{1,5}$, Padmapriya Natarajan ${ }^{3}$, Falk Müller-Riemenschneider ${ }^{2,6}$, Keith M. Godfrey ${ }^{7}$, Peter D. Gluckman ${ }^{1,8}$, Yap-Seng Chong ${ }^{1,3}$, Lynette P. Shek ${ }^{1,4,9}$, Kok Hian Tan ${ }^{10,11}$, Johan G. Eriksson ${ }^{3,12,13,14}$, Fabian Yap ${ }^{11,15,16}$, Yung Seng Lee Le,4,17 $^{1,1}$ and Mary F. F. Chong ${ }^{1,2 *}$

${ }^{1}$ Singapore Institute for Clinical Sciences, Agency for Science, Technology and Research (A*STAR), Singapore 117609

${ }^{2}$ Saw Swee Hock School of Public Health, National University of Singapore, Singapore 117549

${ }^{3}$ Department of Obstetrics \& Gynaecology, Yong Loo Lin School of Medicine, National University of Singapore, Singapore,

Singapore 117597

${ }^{4}$ Department of Pediatrics, Yong Loo Lin School of Medicine, National University of Singapore, Singapore, Singapore 119228

${ }^{5}$ Centre for Research in Epidemiology and StatisticS (CRESS), Université de Paris, Inserm, Inra, F-75004 Paris, France

${ }^{6}$ Institute for Social Medicine, Epidemiology and Health Economics, Charite University Medical Centre, 10117 Berlin, Germany

${ }^{7}$ Medical Research Council Lifecourse Epidemiology Unit and National Institute for Health Research Southampton Biomedical

Research Centre, University of Southampton and University Hospital, Southampton National Health Service Foundation Trust,

Southampton SO16 6YD, UK

${ }^{8}$ Liggins Institute, University of Auckland, Auckland 1023, New Zealand

${ }^{9}$ Divisions of Pediatric Allergy, Immunology, and Rheumatology, Khoo Teck Puat-National University Children's Medical

Institute, National University Hospital, National University Health System, Singapore 119228

${ }^{10}$ Maternal Fetal Medicine, KK Women's and Children's Hospital, Singapore 229899

${ }^{11}$ Duke-National University of Singapore Graduate Medical School, Singapore 169857

${ }^{12}$ Department of General Practice and Primary Health Care, University of Helsinki and Helsinki University Hospital,

FI-OOO14 Helsinki, Finland

${ }^{13}$ Folkhälsan Research Center, FI-O0250 Helsinki, Finland

${ }^{14}$ Department of Chronic Disease Prevention, National Institute for Health and Welfare, FI-00271 Helsinki, Finland

${ }^{15}$ Departments of Paediatrics, KK Women's and Children's Hospital, Singapore 119228

${ }^{16}$ Lee Kong Chian School of Medicine, Nanyang Technological University, Singapore 636921

${ }^{17}$ Division of Paediatric Endocrinology, Khoo Teck Puat-National University Children's Medical Institute, National University Hospital, National University Health System, Singapore 119074

(Submitted 15 January 2019 - Final revision received 31 July 2019 - Accepted 19 August 2019)

\section{Abstract}

Consumption of sugar-sweetened beverages (SSB) by infants and young children are less explored in Asian populations. The Growing Up in Singapore Towards healthy Outcomes cohort study examined associations between SSB intake at 18 months and 5 years of age, with adiposity measures at 6 years of age. We studied Singaporean infants/children with SSB intake assessed by FFQ at 18 months of age ( $n 555)$ and 5 years of age $(n 767)$. The median for SSB intakes is 28 (interquartile range 5.5-98) $\mathrm{ml}$ at 18 months of age and 111 (interquartile range 57-198) $\mathrm{ml}$ at 5 years of age. Association between SSB intake $(100 \mathrm{ml} / \mathrm{d}$ increments and tertile categories) and adiposity measures (BMI standard deviation scores (SD units), sum of skinfolds (SSF)) and overweight/obesity status were examined using multivariable linear and Poisson regression models, respectively. After adjusting for confounders and additionally for energy intake, SSB intake at age 18 months were not significantly associated with later adiposity measures and overweight/obesity outcomes. In contrast, at age 5 years, SSB intake when modelled as $100 \mathrm{ml} / \mathrm{d}$ increments

Abbreviations: GA, gestational age; GUSTO, Growing Up in Singapore Towards healthy Outcomes; IQR, interquartile range; SSB, sugar-sweetened beverages; SSF, sum of skinfolds.

* Corresponding author: Dr Mary F. F. Chong, email mary_chong@nus.edu.sg 
were associated with higher BMI by 0.09 (95\% CI 0.02, 0.16) SD units, higher SSF thickness by 0.68 (95\% CI 0.06, 1.44) mm and increased risk of overweight/obesity by $1.2(95 \%$ CI 1.07, 1.23) times at age 6 years. Trends were consistent with SSB intake modelled as categorical tertiles. In summary, SSB intake in young childhood is associated with higher risks of adiposity and overweight/obesity. Public health policies working to reduce SSB consumption need to focus on prevention programmes targeted at young children.

Key words: Sugar-sweetened beverages: Preschoolers: Infants: Growing Up in Singapore Towards healthy Outcomes: Adiposity: Cohort studies: Asian populations

The prevalence of overweight and obese preschoolers (24-72 months old) in Chinese Singaporeans range from $7 \cdot 0$ to $8 \cdot 1 \%{ }^{(1)}$. These numbers are noteworthy as Southeast Asians face a higher risk of obesity-related disorders such as diabetes, hypertension and CVD that can manifest in children of a younger age compared with ethnic Europeans ${ }^{(2)}$. Furthermore, childhood overweight and obesity tends to be stable (track) through to adulthood $^{(3)}$. One well-documented aspect that may contribute to childhood overweight and obesity risk elsewhere, and now in Southeast Asia, is the increased consumption of soft drinks, juice drinks and other sweetened drinks ${ }^{(4,5)}$.

Early childhood is also the period where food preferences and eating behaviours develop that might serve as the foundation for future eating habits ${ }^{(6)}$. Sugar-sweetened beverage (SSB) consumption patterns in children that start as early as infancy $^{(7,8)}$ and preschool $^{(9)}$ might have lasting implications through childhood, adolescence and into adult years. The negative implications of the overconsumption of SSB on BMI in children aged 6-19 years are well established ${ }^{(4,5)}$. However, the association of SSB consumption with BMI in younger children may not be the same. Cross-sectional studies on SSB intake in preschoolers or younger children have mostly reported positive associations with $\mathrm{BMI}^{(4)}$. However, longitudinal studies relating to SSB intake and BMI present equivocal results: One study reported positive association between SSB consumption in infants 10-12 months of age and their BMI at 6 years ${ }^{(10)}$, while another study in 13-month-olds observed higher BMI in girls, but not in boys at 2, 3, 4 and 6 years of age ${ }^{(8)}$. In children aged $2-5$ years, one study reported a positive association between SSB consumption intake at 2 years of age and higher BMI $z$ score between 2 and 4 years of age ${ }^{(11)}$, while the other study reported no association with BMI 6-12 months later ${ }^{(12)}$.

In the present literature, most studies to date have been conducted in children from Western populations ${ }^{(4)}$. There is a pertinent need to examine this in Asia, especially in Southeast Asia as lifestyles and diets of children here are rapidly changing and becoming increasingly urbanised ${ }^{(13)}$. However, to date, there are only a few studies in Asia itself, and largely confined to older children (6-14 years old). These limited cross-sectional studies in Asia also present conflicting findings. For example, consumption of SSB in two studies from China reported positive associations with obesity in children aged between 3 and 7 years ${ }^{(14)}$ and aged 6 and 13 years ${ }^{(15)}$, but negatively associated with obesity in 9- to 14-year-old Korean boys ${ }^{(16)}$, while a study in 7- to 12 -year-old Taiwanese children found no associations with $\mathrm{BMI}^{(17)}$.

To our knowledge, at present no studies examined SSB consumption patterns in Asian preschoolers or younger children and related them to adiposity outcomes. To address this gap, we used data from a mother-offspring cohort in Singapore which is a microcosm of Asia, which represents three ethnicities (Chinese, Malay and Indians) corresponding to the three major population centres in $\mathrm{Asia}^{(2)}$. We aim to describe the change in absolute amounts of SSB consumed in children aged 18 months and 5 years as part the descriptive analysis of the study and to examine the association of SSB intake at both time points with adiposity measures (BMI $z$ scores and skinfold thickness) and overweight/ obesity status in children at 6 years of age. SSB consumption is hypothesised to be higher in older children, while higher SSB consumption is hypothesised to be associated with higher adiposity measures and risk of overweight/obesity outcomes.

\section{Method \\ Study design}

The Growing Up in Singapore Towards healthy Outcomes (GUSTO) study has been previously described in detail ${ }^{(18)}$. Briefly, GUSTO is a mother-offspring cohort where pregnant women of Chinese, Malay and Indian ethnicities were recruited in their first trimester between June 2009 and September 2010 at two major public maternity units, namely, National University Hospital and KK Women's and Children's Hospital. Of 3751 women screened, 2034 met eligibility criteria ${ }^{(18)}$ and 1247 were recruited into the study. The main exclusion criteria were nonhomogeneous ethnic background (up to the four grandparents of the offspring), intention not to deliver in the study centres or not to remain in Singapore for the following 5 years. The present study was carried out in accordance with the recommendations from the National Healthcare Group Domain Specific Review Board and the SingHealth Centralized Institutional Review Board, with written informed consent from all participants. All participants gave written informed consent in accordance with the Declaration of Helsinki. The protocol was approved by the National Healthcare Group Domain Specific Review Board and the SingHealth Centralized Institutional Review Board (ClinicalTrials.gov; NCT01174875).

At 18 months, there were 555 children with completed FFQ and BMI data; 407 children had completed skinfold thickness data. At age 5 years, there were 767 children with completed FFQ and BMI data; 619 children had completed skinfold thickness data (online Supplementary Fig. S1). A subset of 451 participants with completed FFQ at both time points were used to compare the trends of SSB intake volumes and types consumed and for sensitivity analyses. 
Exposure: dietary assessment at 18 months and 5 years of age

To assess dietary intake at 18 months, the child's primary caregiver (mostly mothers) were given a self-administered 94-item FFQ to complete. At age 5 years, the FFQ was extended to include additional food items that account for greater diet variety at later ages. At this time point, a 125 -item FFQ was administered to primary caregivers by trained interviewers to ascertain the child's diet. The food items in both FFQ were categorised into food groups: (i) bread, (ii) bread spreads, (iii) breakfast cereals, (iv) rice porridge and noodles, (v) potatoes and pasta, (vi) vegetables and bean curd, (vii) fruits, (viii) meat and fish, (ix) eggs, (x) cakes, biscuits and snacks, (xi) milk and dairy products and (xii) other beverages. The FFQ also included general questions on food preparation methods, eating habits and practices and an open-ended section to capture additional food items not listed in the FFQ. Both FFQ were validated either against 24-h recalls at 18 months of age or with 3 -d food diaries at 5 years of age and were previously described in detail. In our validation studies, the correlation analyses of total energy intake were satisfactory at both time points with correlation values of $r 0 \cdot 4^{(19,20)}$. Information for both of the FFQ were collected using the same standardised methods where mothers had to indicate the frequency of consumption over the past month as 'never', 'number of times per month', 'number of times per week' or 'number of times per d'. In addition, they were also asked to indicate a typical serving size for each food consumed, and state the exact amount of beverage consumed in volumes, or units of measure (i.e. cups) or other standard serving sizes (i.e. 1 bottle or 1 packet) which were all converted into volumes of intake (millilitres). Photographs of standardised household measuring utensils and food pictures were presented to assist mothers in quantifying their child's food and beverage intake.

Both the FFQ included seven different beverage items that represent SSB: the frequency of consumption and the volumes consumed per serving were recorded for each item. In the analyses, the definition of SSB included those commonly described in literature $^{(4,5)}$, that is, carbonated, non-carbonated drinks and only prepackaged fruit juices containing added sugar as well as commonly consumed SSB in the Singaporean population such as malted drinks, cultured milk drinks, soya-based drinks and traditional drinks (i.e. barley water, chrysanthemum tea, herbal tea) ${ }^{(21)}$. From the FFQ administered at age 18 months and 5 years, respectively, the total daily intake $(\mathrm{ml} / \mathrm{d})$ of SSB was quantified by multiplying the frequency of consumption per $\mathrm{d}$ by the recorded volume of intake for each specific beverage.

\section{Outcome: anthropometric measures at age 6 years}

At age 6 years, measurements of weight, height and four skinfold thicknesses (triceps, biceps, subscapular and supra-iliac) were obtained by trained staff using standardised protocols as detailed previously ${ }^{(22)}$. Weight was measured to the nearest gram using calibrated SECA 803 weighing scale, and standing height measured using a stadiometer (SECA stadiometer 213). All measurements were taken in duplicate and repeated a third time if there was a difference of $0.2 \mathrm{~kg}$ or $1 \mathrm{~cm}$ for the weight and height measures, respectively. The four skinfolds were measured in triplicate using Holtain skinfold calipers (Holtain Ltd) on the right side of the body and recorded to the nearest $0.2 \mathrm{~mm}$ and repeated a fourth and fifth time if there was a difference of more than $1 \mathrm{~mm}$ between the first three readings. Average values across the repeated measurement were considered for all anthropometry measurements. Four averages were summed to derive the sum of skinfolds (SSF, in $\mathrm{mm}$ ). Sex- and age-specific BMI $z$ scores were calculated using the World Health Organization references ${ }^{(23)}$. The cut-off for overweight and obesity combined were defined as $+1 \mathrm{SD}$ above the reference distribution as per WHO recommendations ${ }^{(24)}$.

\section{Maternal and child covariates}

Maternal socio-demographic data (age, self-reported ethnicity, education level and parity) were obtained using a selfadministered questionnaire at recruitment. Maternal weight and height ( 4 years postpartum) were measured using SECA 803 scales and a SECA 213 stadiometer (SECA Corp.), respectively. These measurements were used to calculate BMI (in $\mathrm{kg} / \mathrm{m}^{2}$ ). Gestational age (GA) was determined by a dating ultrasound scan in the first trimester. At birth and 18 months, weight was measured to the nearest gram (SECA model 334; SECA Corp.), and recumbent length was measured to the nearest $0.5 \mathrm{~cm}$ from the top of the head to the soles of the feet (SECA model 210). The weight of children at 5 years of age was measured to the nearest $10 \mathrm{~g}$ using a calibrated digital scale (SECA model 803; SECACorp.), while standing height was measured using a stadiometer (SECA model 213). For reliability, all measurements were taken in duplicates and averaged. BMI at birth, 18 months and 5 years was calculated as weight divided by the square of recumbent length/height $\left(\mathrm{kg} / \mathrm{m}^{2}\right)$. Skinfold thickness at 18 months and 5 years (triceps, biceps, and subscapular and suprailiac only at 5 years) were obtained to calculate SSF (in $\mathrm{mm})^{(22)}$. We derived birth weightfor-GA and birth length-for-GA $z$ scores using references from our cohort ${ }^{(25)}$. From the obstetric records, we extracted information on child sex. Mothers were asked about the age at which their child had been introduced to solid foods and details on infant milk feeding (as detailed previously) ${ }^{(26)}$ using interviewer-administered infancy questionnaires. The early introduction to solids was defined as the introduction to foods other than milk before the age of 4 months (16 weeks of age) ${ }^{(27)}$. Screen time and outdoor play time were quantified using a parental self-reported interviewer-administered questionnaire when the child turned 3 years of age ${ }^{(28)}$.

\section{Statistical analysis}

The distribution of SSB intake was positively skewed and log transformation could not be performed due to a number of individuals (13\%) with zero intake at 18 months. With that we chose to analyse the SSB intake as (1) a continuous variable modelled as $100 \mathrm{ml}$ increments per $\mathrm{d}$ and (2) a categorical variable of low, medium and high intake based on the tertiles of the study sample at both time points, using separate regression models.

Continuous variables were described as means and standard deviations, and categorical variables as frequencies and percentages. Linear regression models were conducted to examine SSB intake (continuous or categorical) with adiposity outcomes 
(BMI $z$ scores and SSF) at age 6 years. Multivariable Poisson regression with robust variance ${ }^{(29-31)}$ was used to examine SSB intake (continuous or categorical) with the relative risk of being overweight/obese at age 6 years. These were conducted separately for SSB intake at 18 months and 5 years of age. For both the linear and Poisson regression models, the regression coefficient was interpreted as an increase in outcome associated with every $100 \mathrm{ml}$ or with each tertile increase in SSB intake (relative to being in the lowest tertile). Trend tests to test the doseresponse relationship across the tertiles of SSB intake were also performed by modelling the SSB tertiles as a continuous variable. We performed three models: An unadjusted model (model 1), a model adjusted for key potential confounders (model 2) and then additionally adjusted for baseline energy intake respective to the time point of exposure at 18 months or 5 years of age (model 3). The selection of potential confounders (maternal ethnicity, maternal education level, birth weight for GA, parity, breast-feeding duration) was based on the literature ${ }^{(4)}$, or if they changed the effect estimates of our univariate model with tertiles of SSB intake with BMI by $\geq 5 \%{ }^{(32)}$. Screen time at age 3 years and maternal BMI 48 months postpartum were added to the list of confounders when examining the association between SSB intake at age 5 years with adiposity and overweight/obese outcomes.

A sensitivity analysis was conducted to test the robustness of the findings using the subset of 451 participants with completed FFQ at 18 months and 5 years of age. Multiple imputations were used to account for only missing covariate values. Frequencies of missing values were $<5 \%$ for breast-feeding duration and maternal education, and slightly higher for child screen time and maternal postpartum BMI (12-18\%). All values were assumed to be missing at random based on the Little Missing Completely at Random test $(P$ value $>0 \cdot 05)$. Missing values were imputed twenty times using multiple imputation analysis and chain equations. Imputations were based on available information on all exposure and outcome variables included in the study. Analyses were performed in each of the imputed data sets and the final results were pooled. We reported association estimates with their $95 \%$ CI. All analyses were performed using SPSS IBM version 20 .

\section{Results}

\section{Characteristics of mothers and children}

Among the 767 participants who completed the FFQ at age 5 years, $98 \%$ reported their child consuming some form of SSB. Children in the high intake tertile (median 241 (interquartile range (IQR) 197-328) $\mathrm{ml}$ ) had mothers who were more likely to be of Malay ethnicity, had lower educational attainment, were multiparous and had higher maternal postpartum BMI, compared with those in the low ( 40 (IQR 21-57) ml) and middle (111 (IQR 93-137) ml) tertiles. Additionally, these children were most likely to have more than $4 \mathrm{~h}$ of screen time viewing per $\mathrm{d}$ at age 3 years and had the highest total energy intake, BMI $z$ scores and SSF at age 5 years (Table 1$)$. The characteristics of participants who were $(n$ 767) and were not $(n 320)$ included in the analyses were similar except for maternal age (included mothers were slightly older) (online Supplementary Table S1). All maternal and infant characteristics significantly associated with SSB intake tertiles at age 5 years were largely similar to SSB intake at age 18 months in 555 participants, except maternal parity, child screen time, BMI $z$ scores and skinfolds where no significant differences were observed with SSB intake tertiles at 18 months. While there was no significant difference between the intake tertiles at age 5 years with maternal age, higher SSB intake tertiles at 18 months were associated with younger mothers. Furthermore, the volume of intake in each tertile at age 18 months was lower compared with that at age 5 years (online Supplementary Table S2).

\section{Trends in sugar-sweetened beverage intake from age 18 months to 5 years}

In the subset of 451 children with data at both the 18 month and age 5 year time points, SSB intakes were compared. Overall, a higher proportion of participants were consuming higher volumes of SSB $(50->400 \mathrm{ml} v .0-50 \mathrm{ml})$ at age 5 years $(5 \cdot 8-32 \cdot 4 \%)$ compared with 18 months of age (3.3-13.5\%) (Fig. 1). Furthermore, a higher proportion of children at age 5 years were consuming malted, cultured and sweetened drinks, but fewer consumed soya milk and traditional herbal tea compared with children aged 18 months $(P<0 \cdot 05)$ (Fig. 2$)$.

Sugar-sweetened beverage intake at age 18 months with $B M I$ z score, sum of skinfold thickness and overweight/ obesity at age 6 years

SSB intake at 18 months modelled as $100 \mathrm{ml} / \mathrm{d}$ increments were associated only with higher risk of overweight/obesity (relative risk 1.09; $95 \%$ CI 1.02, 1.64), but not with BMI $z$ scores or SSF. However, after further adjustment for energy intake, this association was no longer significant (Table 2). There were also no significant association between the medium/high SSB intake tertiles at age 18 months for both adiposity outcomes (BMI $z$ score and SSF thickness) and risk of overweight/obesity at age 6 years, when compared with the lowest intake tertile $\left(P_{\text {trend }}>0 \cdot 05\right)$. These findings remained even after further adjustment for energy intake (Table 2), and in the sensitivity analysis of 451 children (online Supplementary Table S3).

Sugar-sweetened beverage intake at age 5 years with BMI z score, sum of skinfold thickness and overweight/obesity at age 6 years

Higher volumes of SSB (per $100 \mathrm{ml}$ increment) were associated with higher BMI by 0.09 (95\% CI 0.03, 0.15) sD units, higher SSF thickness by $0.75(95 \%$ CI $0.06,1.44) \mathrm{mm}$ and increased risk of overweight/obesity by $1.2(95 \%$ CI $1.07,1.23)$ times at age 6 years. Similar trends were seen with the categories of SSB intake, with the high SSB intake tertile being significantly associated with higher BMI $z$ scores by 0.33 (95\% CI $0 \cdot 11,0 \cdot 55$ ) SD units, and higher risk of overweight/obesity (relative risk 1.56; $95 \%$ CI 1.05, 2.33) at age 6 years when compared with the low intake tertile $\left(P_{\text {for trend }}<0 \cdot 05\right)$. No significant associations were found between the medium and high SSB intake tertiles and SSF 
Table 1. Maternal and child's characteristics according to tertiles of sugar sweetened beverage (SSB) intake at 5 years of age $(n 767)^{\star} \dagger$ (Numbers and percentages; mean values and standard deviations)

\begin{tabular}{|c|c|c|c|c|c|c|c|}
\hline & \multicolumn{2}{|c|}{$\begin{array}{c}\text { Low intake: } \\
40(21-57 \mathrm{ml}) \ddagger(n \text { 255) }\end{array}$} & \multicolumn{2}{|c|}{$\begin{array}{c}\text { Medium intake: } \\
111(93-137 \mathrm{ml}) \neq(n \text { 256) }\end{array}$} & \multicolumn{2}{|c|}{$\begin{array}{c}\text { High intake: } \\
241(197-328 \mathrm{ml}) \ddagger \\
(n \text { 256) }\end{array}$} & \multirow[b]{2}{*}{$P \S$} \\
\hline & $n$ & $\%$ & $n$ & $\%$ & $n$ & $\%$ & \\
\hline Maternal ethnicity & & & & & & & $<0.0001$ \\
\hline Chinese & 161 & 63.1 & 154 & $60 \cdot 2$ & 118 & $46 \cdot 1$ & \\
\hline Malay & 44 & $17 \cdot 3$ & 61 & $23 \cdot 8$ & 87 & $34 \cdot 0$ & \\
\hline Indian & 50 & $19 \cdot 6$ & 41 & $16 \cdot 0$ & 51 & $19 \cdot 9$ & \\
\hline Maternal educational level & & & & & & & 0.016 \\
\hline Post-secondary and below & 146 & 57.5 & 172 & $67 \cdot 7$ & 173 & 63.4 & \\
\hline University and above & 108 & $42 \cdot 5$ & 82 & $32 \cdot 3$ & 80 & $31 \cdot 6$ & \\
\hline Parity & & & & & & & 0.001 \\
\hline Primiparous & 126 & $49 \cdot 4$ & 131 & $51 \cdot 2$ & 91 & 35.5 & \\
\hline Multiparous & 129 & $50 \cdot 6$ & 125 & $48 \cdot 8$ & 165 & 64.5 & \\
\hline Maternal age at first birth (years) & & & & & & & 0.193 \\
\hline Mean & \multicolumn{2}{|c|}{$31 \cdot 3$} & \multicolumn{2}{|c|}{$30 \cdot 9$} & \multicolumn{2}{|c|}{$30 \cdot 7$} & \\
\hline SD & \multicolumn{2}{|c|}{$5 \cdot 1$} & \multirow{2}{*}{\multicolumn{2}{|c|}{$5 \cdot 1$}} & \multicolumn{2}{|c|}{5.4} & \\
\hline Postpartum BMI at 4 years $\left(\mathrm{kg} / \mathrm{m}^{2}\right)$ & & & & & & & 0.010 \\
\hline Mean & \multicolumn{2}{|c|}{23.8} & \multicolumn{2}{|c|}{$24 \cdot 9$} & \multicolumn{2}{|c|}{$25 \cdot 1$} & \\
\hline SD & \multicolumn{2}{|c|}{4.7} & \multicolumn{2}{|c|}{$5 \cdot 8$} & \multicolumn{2}{|c|}{4.9} & \\
\hline Child sex & & & & & & & 0.332 \\
\hline Boy & 125 & $49 \cdot 0$ & 131 & $51 \cdot 2$ & 142 & 55.5 & \\
\hline Girl & 130 & 51.0 & 125 & $48 \cdot 8$ & 114 & 44.4 & \\
\hline Breast-feeding status & & & & & & & 0.695 \\
\hline Never breast-fed & 11 & 4.4 & 10 & 4.0 & 13 & 5.3 & \\
\hline Breast-fed for $<6$ months & 207 & 82.5 & 216 & 86.4 & 207 & $84 \cdot 1$ & \\
\hline Breast-fed for $\geq 6$ months & 33 & $13 \cdot 1$ & 24 & $9 \cdot 6$ & 26 & $10 \cdot 6$ & \\
\hline Early introduction to solids ( $<16$ weeks of age) $\|$ & & & & & & & 0.143 \\
\hline Yes & 227 & $97 \cdot 4$ & 229 & 99.5 & 224 & 97.4 & \\
\hline No & 6 & $2 \cdot 6$ & 1 & 0.5 & 6 & $2 \cdot 6$ & \\
\hline Child screen time $(\mathrm{h} / \mathrm{d})$ & & & & & & & 0.012 \\
\hline$<2$ & 117 & 51.5 & 92 & 41.4 & 82 & 35.5 & \\
\hline $2-4$ & 64 & $28 \cdot 2$ & 77 & 34.7 & 82 & 35.5 & \\
\hline$>4$ & 46 & $20 \cdot 3$ & 53 & 23.9 & 67 & $29 \cdot 0$ & \\
\hline Child outdoor playing $(\mathrm{h} / \mathrm{d})$ & & & & & & & 0.776 \\
\hline$<2$ & 191 & $86 \cdot 8$ & 186 & 89.4 & 184 & 85.6 & \\
\hline $2-4$ & 28 & $12 \cdot 7$ & 21 & $10 \cdot 1$ & 29 & 13.5 & \\
\hline$>4$ & 1 & 0.5 & 1 & 0.5 & 2 & 0.9 & \\
\hline Birth weight for gestational age ( $z$ scores) & & & & & & & 0.115 \\
\hline Mean & & & & & & & \\
\hline SD & & & & & & & \\
\hline Child energy intake at 5 years & & & & & & & $<0.0001$ \\
\hline $\mathrm{kcal} / \mathrm{d}$ & & & & & & & \\
\hline Mean & & & & & & & \\
\hline SD & & & & & & & \\
\hline $\mathrm{kJ} / \mathrm{d}$ & & & & & & & \\
\hline Mean & & & & & & & \\
\hline SD & & & & & & & \\
\hline BMI at 18 months ( $z$ scores) & & & & & & & 0.259 \\
\hline Mean & & & & & & & \\
\hline SD & & & & & & & \\
\hline$\Sigma$ Skinfolds at 18 months $(\mathrm{mm})$ & & & & & & & 0.276 \\
\hline Mean & & & & & & & \\
\hline SD & & & & & & & \\
\hline BMl at 5 years ( $z$ scores) & & & & & & & $<0.0001$ \\
\hline Mean & & & & & & & \\
\hline SD & & & & & & & \\
\hline$\Sigma$ Skinfolds at 5 years $(\mathrm{mm})$ & & & & & & & 0.013 \\
\hline Mean & & & & & & & \\
\hline SD & & & & & & & \\
\hline
\end{tabular}

GUSTO, Growing Up in Singapore Towards healthy Outcomes.

* Data were missing for education ( $n 6)$, breast-feeding ( $n$ 20), maternal postpartum BMl at 48 months ( $n$ 97), birth weight for gestational age ( $n$ 12), early introduction to solid foods $(n 74)$, sum of skinfolds ( $n$ 148), screen time $(n 87)$ and outdoor playing $(n 124)$.

$\dagger P$ value across the SSB tertile categories was determined with the use of a $\chi^{2}$ analysis (categorical) or trend tests using SSB intake tertile categories as continuous variable (continuous).

$\ddagger$ Values reflect medians and interquartile ranges.

$\S P<0.05$ is statistically significant.

|| Early introduction to solids was defined as introduction to foods other than milk before the age of 4 months (16 weeks of age). 


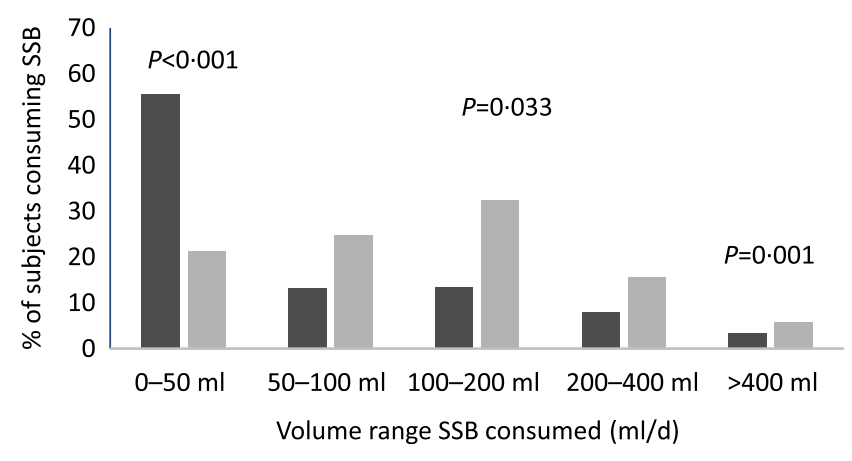

Fig. 1. Proportion of children consuming sugar-sweetened beverages (SSB) at 18 months $(\square)$ and 5 years $(\square)$ of age by volume range $(n 451)$. To determine the differences in the proportion of participants consuming and not consuming SSB for each specific volume range, $\chi^{2}$ analysis was used.

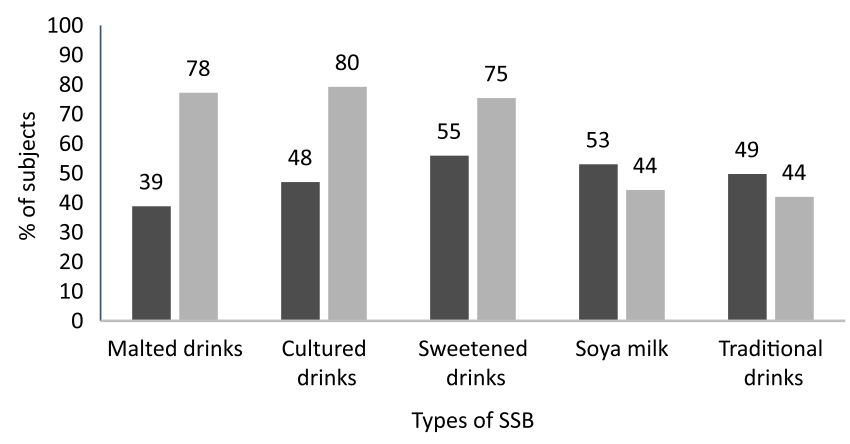

Fig. 2. Types of sugar-sweetened beverages (SSB) consumed at age 5 years ( $)$ compared with 18 months $(\square)$ (subset of $n$ 451). There were statistically significant differences between the proportion of children at 18 months and 5 years for all SSB types $\left(P<0.05 ; \chi^{2}\right.$ analysis $)$.

thickness $\left(P_{\text {for trend }}>0 \cdot 05\right)$. All associations remained even after further adjustment for energy intake (Table 3).

In sensitivity analyses ( $n$ 451), similar associations were observed between the volumes of SSB (per $100 \mathrm{ml}$ increment) with all outcomes. However, the results from the associations between the SSB intake tertiles and BMI $z$ scores and SSF thickness differed in this subset: Both the medium and high intake tertiles were associated with higher BMI $z$ scores $(\beta 0.34(95 \%$ CI $0 \cdot 06,0 \cdot 62)$ sD units and $\beta 0 \cdot 46(95 \% \mathrm{CI} 0 \cdot 18,0 \cdot 74)$ SD units, respectively). Furthermore, the high intake tertile was associated with higher skinfold measures by $3 \cdot 25$ (95\% CI 0.36, 6.14) $\mathrm{mm}\left(P_{\text {for }}\right.$ trend $<0.05)$ which was previously not observed in the 767 participants. All associations remained after further adjustment for energy intake (online Supplementary Table S4).

\section{Discussion}

Our study is the first prospective Asian Singaporean cohort study to observe that the consumption of SSB in preschoolers (age 5 years) and young (18 months) children is associated with later childhood adiposity measures (BMI and skinfold thickness) and overweight/obesity outcomes at age 6 years. At age 5 years, every $100 \mathrm{ml} / \mathrm{d}$ increment of SSB intake was associated with 0.09 SD unit higher BMI and $0.8 \mathrm{~mm}$ higher SSF thickness, and $1 \cdot 2$-fold higher risk of overweight/obesity at age 6 years.
Similarly, children in the highest intake tertile (median intake $=$ approximately $250 \mathrm{ml}$ ) of SSB consumption at this age had a $0 \cdot 3$ SD unit higher BMI, and almost a 1.6-fold higher risk of overweight/obesity at age 6 years, compared with those in the low intake tertile (median intake $=$ approximately $50 \mathrm{ml} / \mathrm{d}$ ), and there were positive non-significant trends with SSF thickness. All the associations were still significant after the adjustment for energy intake. In contrast, there were overall null findings between the SSB intake at 18 months with child adiposity at age 6 years. Few studies in 1 - to 2 -year-old children have observed positive association between SSB intake and adiposity outcomes. A small sample of 97 Latino mother and toddler pairs aged between 1 and 2 years observed that higher consumption of SSB above the median was associated with higher BMI after a 6-month follow-up ${ }^{(33)}$. Two studies in the USA examined SSB intake in 2-year-olds, with one reporting that children drinking SSB (compared with infrequent/no drinkers) had higher BMI at 4 years of age ${ }^{(11)}$, while another reported that high intake ( $>3$ times/week) $v$. no intake of SSB was associated with higher odds of developing obesity at 6 years of age ${ }^{(10)}$. Lastly, high intake $(15$ servings $/$ week $=320 \mathrm{ml} / \mathrm{d}$ ) of SSB in the 13 -monthold Dutch toddlers were significantly associated with increased BMI but only in girls up to 6 years of age ${ }^{(8)}$. The overall null associations we observed with SSB intake at 18 months could be attributed to the lower volumes of SSB consumed at this time point by the children in our cohort (the median volume in our high intake tertile was only 138 (IQR 98-231) ml/d; the low volume was only 3 (IQR 0-7) $\mathrm{ml} / \mathrm{d}$ ). Alternatively, another potential concern regarding SSB intake at 18 months is the use of the maternal report of dietary assessments which was self-administered at this time point. This will inevitably include some degree of measurement errors due to recall bias or improper reporting that may have attenuated true associations in the present study. With the exception of the study in Dutch toddlers mentioned earlier ${ }^{(8)}$, comparisons of the intake volumes across the studies in children within a similar age group is difficult as typical serving sizes of SSB (in $\mathrm{ml} / \mathrm{d}$ ) are usually not ascertained, or different dietary assessment methods have been used (e.g. 24-h recall/ interview questionnaire $v$. an FFQ $)^{(10,11,33)}$.

Our findings related to children's SSB intake at 5 years, with BMI and overweight/obesity outcomes consistent across the different measures, and the association remained significant even in a subset analysis. These observations concur with the few existing cross-sectional studies ${ }^{(34-36)}$ and one prospective study $^{(11)}$ in children of a similar age range. These studies have all used body weight measures (BMI or BMI $z$ score) as a main outcome and have defined the overweight/obesity statuses of children $^{(11,14,34,35)}$, but none examined SSB intake with skinfold measures. SSF thickness (usually biceps, triceps, supra-iliac and subscapular) has been shown to be a useful supplementary measure widely used to assess body fatness in children ${ }^{(37)}$. In our study, skinfold thickness was only significantly associated with adiposity outcomes when SSB intake at 5 years were modelled as $100 \mathrm{ml} / \mathrm{d}$ increments, but not with the tertile intake, which could be due to the loss of power from the categorisation into tertile groups ${ }^{(38)}$.

The underlying biological mechanism by which SSB intake affects adiposity has not been fully elucidated ${ }^{(39)}$. 
Table 2. Associations between sugar-sweetened beverage (SSB) consumption at 18 months with adiposity measures at 6 years ( $\beta$-Coefficients or relative risk (RR) and $95 \%$ confidence intervals)

\begin{tabular}{|c|c|c|c|c|c|c|}
\hline \multirow[b]{2}{*}{ SSB intake at 18 months } & \multicolumn{2}{|c|}{ BMI $z$ scorest‡ ( $n$ 555) } & \multicolumn{2}{|c|}{ SSF†§ $(n$ 407) } & \multicolumn{2}{|c|}{$\begin{array}{l}\text { Overweight/obesity } \ddagger \| \\
\text { (n 555) }\end{array}$} \\
\hline & $\beta$ & $95 \% \mathrm{Cl}$ & $\beta$ & $95 \% \mathrm{Cl}$ & $\mathrm{RR}$ & $95 \% \mathrm{Cl}$ \\
\hline \multicolumn{7}{|l|}{ Model 1 (unadjusted) } \\
\hline $100 \mathrm{ml} / \mathrm{d}$ increments & $0.09^{*}$ & $0.03,0.15$ & $1.04^{*}$ & $0.30,1.77$ & $1 \cdot 10^{\star}$ & $1.03,1.17$ \\
\hline Low intake & \multicolumn{2}{|c|}{ Reference } & \multicolumn{2}{|c|}{ Reference } & \multicolumn{2}{|c|}{ Reference } \\
\hline Medium intake & -0.04 & $-0.29,0.22$ & -0.62 & $-3 \cdot 38,2 \cdot 14$ & 0.92 & $0.54,1.56$ \\
\hline High intake & 0.23 & $-0.02,0.48$ & 0.96 & $-1 \cdot 80,3 \cdot 72$ & 1.40 & $0 \cdot 87,2 \cdot 24$ \\
\hline $\begin{array}{l}P_{\text {trend }} \\
\text { Model } 2 \text { (adjusted) }\end{array}$ & \multicolumn{2}{|c|}{$P=0.066$} & \multicolumn{2}{|c|}{$P=0.493$} & \multicolumn{2}{|c|}{$P=0.071$} \\
\hline $100 \mathrm{ml} / \mathrm{d}$ increments & 0.06 & $-0.002,0.12$ & 0.68 & $-0.05,1.42$ & $1.09^{*}$ & $1.02,1.64$ \\
\hline Low intake & \multicolumn{2}{|c|}{ Reference } & \multicolumn{2}{|c|}{ Reference } & \multicolumn{2}{|c|}{ Reference } \\
\hline Medium intake & -0.05 & $-0.29,0.20$ & -0.91 & $-3.57,1.76$ & 0.93 & $0.55,1.58$ \\
\hline High intake & 0.08 & $-0.17,0.33$ & -0.42 & $-3 \cdot 18,2 \cdot 35$ & $1 \cdot 12$ & $0 \cdot 68,1 \cdot 84$ \\
\hline $\begin{array}{l}P_{\text {trend }} \\
\text { Model } 3 \text { (energy intake adjusted) }\end{array}$ & \multicolumn{2}{|c|}{$P=0.532$} & \multicolumn{2}{|c|}{$P=0.758$} & \multicolumn{2}{|c|}{$P=0.194$} \\
\hline $100 \mathrm{ml} / \mathrm{d}$ increments & 0.05 & $-0.01,0.11$ & 0.71 & $-0.05,1.47$ & $1.09^{*}$ & $\begin{array}{l}1.02,1 \cdot 17 \\
n c e\end{array}$ \\
\hline Low intake & \multicolumn{2}{|c|}{ Reference } & \multicolumn{2}{|c|}{ Reference } & \multicolumn{2}{|c|}{ Reference } \\
\hline Medium intake & -0.05 & $-0.29,0.19$ & -0.92 & $-3 \cdot 59,1 \cdot 75$ & 0.93 & $0.55,1.58$ \\
\hline High intake & 0.06 & $-0.20,0.31$ & -0.46 & $-3 \cdot 27,2 \cdot 34$ & $1 \cdot 10$ & $0 \cdot 67,1 \cdot 81$ \\
\hline$P_{\text {trend }}$ & \multicolumn{2}{|c|}{$P=0.676$} & \multicolumn{2}{|c|}{$P=0.850$} & \multicolumn{2}{|c|}{$P=0.204$} \\
\hline
\end{tabular}

SSF, sum of skinfolds; IQR, interquartile range.

${ }^{*} P<0.05$.

$\dagger$ Estimated regression coefficients.

‡ Median, $n$ : low intake 2 (IQR 0-6 ml), $n$ 185; medium intake 28 (IQR 18-43 ml), $n$ 185; high intake 138 (IQR 98-231 ml), $n 185$.

$\S$ Median, $n$ : low intake 3 (IQR 0-7 ml), $n$ 135; medium intake 28 (IQR 18-43 ml), $n$ 136; high intake 136 (IQR 99-238 ml), $n 136$.

$\| 95 \% \mathrm{Cl}$ of the association between SSB intake (high and medium compared with low as reference) with BMI $z$ scores and overweight/obesity outcomes at 6 years of age. Trend tests were performed using categories of SSB intake as continuous variable in the linear regression and Poisson regression models. Models are adjusted for ethnicity, education, birth weight for gestational age, screen time, breast-feeding duration and parity. Model 3 is model 2 additionally adjusted for energy intake at age 18 months.

Table 3. Associations between sugar-sweetened beverage (SSB) consumption at 5 years with adiposity measures at 6 years ( $\beta$-Coefficients or relative risk (RR) and $95 \%$ confidence intervals)

\begin{tabular}{|c|c|c|c|c|c|c|}
\hline \multirow[b]{2}{*}{ SSB intake at 5 years } & \multicolumn{2}{|c|}{ BMI $z$ scorest‡ ( $n$ 767) } & \multicolumn{2}{|c|}{ SSF†§ ( $n 619)$} & \multicolumn{2}{|c|}{$\begin{array}{l}\text { Overweight/obesity } \ddagger \| \\
(n \text { 767) }\end{array}$} \\
\hline & $\beta$ & $95 \% \mathrm{Cl}$ & $\beta$ & $95 \% \mathrm{Cl}$ & $\mathrm{RR}$ & $95 \% \mathrm{Cl}$ \\
\hline \multicolumn{7}{|l|}{ Model 1 (unadjusted) } \\
\hline $100 \mathrm{ml} / \mathrm{d}$ increments & $0.12^{\star \star}$ & $0.05,0.18$ & $0.83^{*}$ & $0.15,1.52$ & $1 \cdot 16^{\star *}$ & $1.09,1.23$ \\
\hline Low intake & \multicolumn{2}{|c|}{ Reference } & \multicolumn{2}{|c|}{ Reference } & \multicolumn{2}{|c|}{ Reference } \\
\hline Medium intake & $0.23^{*}$ & $0.01,0.46$ & $1 \cdot 25$ & $-1 \cdot 19,3.69$ & 1.25 & $0.78,1.98$ \\
\hline High intake & $0.42^{\star *}$ & $0.20,0.65$ & $2 \cdot 62^{*}$ & $0.17,5.06$ & $1 \cdot 74^{\star}$ & $1.13,2.69$ \\
\hline$P_{\text {trend }}$ & \multicolumn{2}{|c|}{$P<0.001$} & \multicolumn{2}{|c|}{$P=0.036^{\star}$} & \multirow{2}{*}{\multicolumn{2}{|c|}{$P=0.005$}} \\
\hline Model 2 (adjusted) & & & & & & \\
\hline $100 \mathrm{ml} / \mathrm{d}$ increments & $0.09^{*}$ & $0.03,0.15$ & $0.75^{\star}$ & $0.06,1.44$ & $1 \cdot 15^{\star *}$ & $1.07,1.23$ \\
\hline Low intake & \multicolumn{2}{|c|}{ Reference } & \multicolumn{2}{|c|}{ Reference } & \multicolumn{2}{|c|}{ Reference } \\
\hline Medium intake & 0.17 & $-0.05,0.38$ & 0.53 & $-1 \cdot 89,2.94$ & $1 \cdot 13$ & $0.74,1.71$ \\
\hline High intake & $0.33^{*}$ & $0.11,0.55$ & 1.93 & $-0.52,4.37$ & $1.56^{\star}$ & $1.05,2.33$ \\
\hline $\begin{array}{l}P_{\text {trend }} \\
\text { Model } 3 \text { (energy intake adjusted) }\end{array}$ & \multicolumn{2}{|c|}{$P=0.003$} & \multicolumn{2}{|c|}{$P=0.123$} & \multicolumn{2}{|c|}{$P=0.025$} \\
\hline $100 \mathrm{ml} / \mathrm{d}$ increments & $0.09^{*}$ & $0.02,0.16$ & $0.87^{\star}$ & $0.14,1.60$ & $1 \cdot 15^{\star}$ & $1 \cdot 06,1.24$ \\
\hline Low intake & \multicolumn{2}{|c|}{ Reference } & \multicolumn{2}{|c|}{ Reference } & \multicolumn{2}{|c|}{ Reference } \\
\hline Medium intake & 0.17 & $-0.05,0.39$ & 0.62 & $-1.81,3.04$ & $1 \cdot 12$ & $0.74,1.69$ \\
\hline High intake & $0.34^{*}$ & $0.11,0.58$ & $2 \cdot 20$ & $-0.38,4.78$ & $1.54^{\star}$ & $1.03,2 \cdot 30$ \\
\hline$P_{\text {trend }}$ & \multicolumn{2}{|c|}{$P=0.004$} & \multicolumn{2}{|c|}{$P=0.096$} & \multicolumn{2}{|c|}{$P=0.033$} \\
\hline
\end{tabular}

SSF, sum of skinfolds; IQR, interquartile range.

${ }^{\star} P<0.05,{ }^{* *} P<0.01$.

† Estimated regression coefficients.

‡ Median, $n$ : low intake 40 (IQR 21-57 ml), $n$ 255; medium intake 111 (IQR 93-137 ml), $n$ 256; high intake 241 (IQR $197-328 \mathrm{ml}), n 256$

$\S$ Median, $n$ : low intake 37 (IQR 21-54 ml), $n$ 202; medium intake 108 (IQR 91-134 ml), $n$ 209; high intake 241 (IQR $195-317 \mathrm{ml}$ ), $n 208$.

$\| 95 \% \mathrm{Cl}$ of the associations between SSB intake tertiles (high and medium with low as reference) with BMI $z$ scores and overweight/obesity outcomes at 6 years of age. Trend tests were performed using categories of SSB intake as continuous variable in the linear regression and Poisson regression models. Models are adjusted for ethnicity, education, birth weight for gestational age, screen time, breast-feeding duration, maternal BMI 48 months postpartum and parity, SSB intake at 18 months. Model 3 is model 2 additionally adjusted for energy intake at age 5 years. 
The hypothesised mechanism for the potential causal relationship between SSB intake and obesity is thought to be mediated through the increase in energy intake resulting from the reduction in satiety and incomplete energetic compensation at subsequent meals ${ }^{(5)}$. However, the additional adjustment for total energy intake in our regression models did not affect most of the estimated significant association in our main data set as well as in our subset analysis. Similar observations were seen in previous studies ${ }^{(8,39,40)}$, suggesting that the associations may be explained by non-energy effects of SSB. High intake of SSB in preschoolers could be just markers of poor diet quality where these children are consuming high amounts of sweet foods, along with high intake of sugary beverages ${ }^{(41)}$. High glycaemic loads from the possible high sugar diet alone could induce hyperinsulinaemia, leading to increase in fat deposition that is independent of energy intake ${ }^{(42)}$. Another alternate mechanism linking poor diet and obesity could be through the mediation effects of the microbiome profile in the gut. Poorer diet quality (e.g. Western dietary patterns of high-fat food and refined sugars) has been associated with a specific diversity of microbiota termed the 'obesogenic microbiota', which has been shown to display enhanced capacity for energy harvest from the diet that might lead to weight gain ${ }^{(43)}$. Overall, poorer diet quality that might lead to higher glycaemic loads and the presence of obesogenic microbiota in the gut are all possible contributing factors to the non-energy effects between SSB and adiposity.

In our present findings, we found a trend of higher volumes of SSB consumption in older children of preschool age ( 5 years $v$. 18 months). In our study, $98 \%$ of participants were consuming SSB by 5 years of age, and this percentage is higher than the $80.5 \%$ children in China consuming SSB by 3-7 years of age ${ }^{(14)}$. This observation is in line with a survey conducted in 800 Singaporean children in 2009 examining their beverage consumption habits. The survey showed that consumption of SSB increased with age (7-10 years $v$. 3-6 years ${ }^{(21)}$. Higher consumptions of SSB were also observed to be displacing the consumption of healthier and more nutrient-dense beverages like milk ${ }^{(21)}$. Furthermore, the differences in the types of SSB consumed between the two age groups (18 months $v .5$ years) in the present study suggest that age-relevant interventions are needed to target the main types consumed. This may have implications on the current daily recommendations for sugar intake in Singaporean toddlers and preschoolers ${ }^{(44)}$.

Strengths of the present study are the prospective data collection (1-year follow-up) and assessment of a wide range of socio-demographic and lifestyle factors that were adjusted for in multivariable analyses. Although we only had FFQ data at 5 years for $71 \%$ of the mother-child dyads who participated at the start, no differences were observed between the participants who were excluded and included in the study except for maternal age. We analysed a number of adiposity measures, including SSF thickness measures. Several limitations of the present study need to be acknowledged. Using an FFQ to assess dietary intake is the most appropriate tool because it provides an advantage over a single 24-h dietary recall or food diaries by capturing habitual intake (over $30 \mathrm{~d}$ ) ${ }^{(45)}$. However, like all dietary assessments, measurement errors in dietary intake may still be present ${ }^{(46)}$. For example, mothers whose children attending day care on weekdays might be less aware of their children's exact food intake and are thus less likely to report their daily intake accurately. Since this FFQ captured maternal self-reported dietary intake of children, a systematic bias might occur if mothers underreport intake of SSB to meet more socially desirable intake ${ }^{(47)}$. However, we attempted to control for this using cut-offs within the 500-4000 kcal range for energy intake, suitable for children of this age range to identify dietary underreporters and over-reporters ${ }^{(48)}$. Because of the observational design of our study, casual effects of SSB on child adiposity could not be determined. Furthermore, the relatively short follow-up (1 year) between SSB intake at age 5 years and adiposity outcomes at age 6 years might not be sufficient for us to observe longer lasting effects of SSB on adiposity, and because of this short follow-up, we cannot rule out the possibility of reverse causation (i.e. higher BMI or energy intake leading to higher consumptions of SSB). Although we collected data on child outdoor activity, the self-reported method used to quantify physical activity in the present study can underestimate the strength of the relationship between this covariate and the risk factor of interest (SSB intake). Future studies will benefit from objective physical activity data measured with an accelerometer rather than questionnairebased activity measures. Lastly, although we attempted to control for major confounders in the present study, residual confounding (i.e. other lifestyle factors such as diet quality) could not be ruled out.

\section{Conclusion}

In conclusion, our observations suggest that SSB consumption should be limited in young children to address the raising prevalence of child obesity. It also further contributes to the emerging consensus of the negative effects of SSB consumption in children below school age. The development of public health strategies to educate childcare centres and parents of toddlers and preschoolers about the health implications of high consumption of SSB should be a priority. Finally, further longitudinal analyses are needed to better understand the mechanisms involved and the effects of SSB intake on long-term weight gain.

\section{Acknowledgements}

We thank the members of the GUSTO study group: Allan Sheppard, Amutha Chinnadurai, Anne Eng Neo Goh, Anne Rifkin-Graboi, Anqi Qiu, Arijit Biswas, Bee Wah Lee, Birit F. P. Broekman, Boon Long Quah, Borys Shuter, Carolina Un Lam, Chai Kiat Chng, Cheryl Ngo, Choon Looi Bong, Christiani Jeyakumar Henry, Claudia Chi, Cornelia Yin Ing Chee, Yam Thiam Daniel Goh, Doris Fok, E. Shyong Tai, Elaine Tham, Evelyn Xiu Ling Loo, Fabian Yap, Falk MuellerRiemenschneider, George Seow Heong Yeo, Helen Chen, Heng Hao Tan, Hugo P. S. van Bever, Iliana Magiati, Inez Bik Yun Wong, Ivy Yee-Man Lau, Jeeves Kapur, Jenny L. Richmond, Jerry Kok Yen Chan, Joanna D. Holbrook, Joanne Yoong, Joao N. Ferreira, Jonathan Tze Liang Choo, Jonathan Y. Bernard, Joshua J. Gooley, Kenneth Kwek, Krishnamurthy Niduvaje, Kuan Jin Lee, Leher Singh, Lieng Hsi Ling, Lin Lin Su, Ling-Wei Chen, Lourdes Mary Daniel, Marielle V. Fortier, Mark 
Hanson, Mary Rauff, Mei Chien Chua, Melvin Khee-Shing Leow, Michael Meaney, Mya Thway Tint, Neerja Karnani, Ngee Lek, Oon Hoe Teoh, P. C. Wong, Paulin Tay Straughan, Pranitha Agarwal, Queenie Ling Jun Li, Rob M. van Dam, Salome A. Rebello, SeangMei Saw, See Ling Loy, S. Sendhil Velan, Seng Bin Ang, Shang Chee Chong, Sharon Ng, Shiao-Yng Chan, Shirong Cai, Shu-E Soh, Sok Bee Lim, Stella Tsotsi, Chin-Ying Stephen Hsu, Sue Anne Toh, Swee Chye Quek, Victor Samuel Rajadurai, Walter Stunkel, Wayne Cutfield, Wee Meng Han, Wei Wei Pang, Yin Bun Cheung, Yiong Huak Chan and Zhongwei Huang.

This research is supported by the Singapore National Research Foundation under its Translational and Clinical Research (TCR) Flagship Programme and administered by the Singapore Ministry of Health's National Medical Research Council (NMRC), Singapore- NMRC/TCR/004-NUS/2008; NMRC/TCR/012-NUHS/ 2014. Additional funding is provided by the Singapore Institute for Clinical Sciences, Agency for Science, Technology and Research (A*STAR), Singapore. K. M. G. is supported by the National Institute for Health Research through the NIHR Southampton Biomedical Research Centre and by the European Union's Erasmus+ Capacity-Building ENeA $^{\text {SEA }}$ Project and Seventh Framework Program (FP7/2007-2013), projects Early Nutrition and ODIN under grant agreement numbers 289346 and 613977. Additional funding of the present study was provided by the Singapore Institute for Clinical Sciences, A*STAR.

F. Y., K. M. G., Y.-S. C., L. P. S. and K. H. T. designed and led the GUSTO cohort study. P. L. Q., J. K. and Y. Y. C. contributed to the statistical analysis and writing of the manuscript. J. Y. T. cleaned and processed the data for SSB intake. H. X. L. and R. S. cleaned, processed and performed the validation of the FFQ at 18 months and 5 years of age. J. Y. B. and P.N. cleaned the data set for screen time and outdoor play. I. M. A., W. L. Y., M. T. T. and Y. S. L. contributed to the collection and processing of the anthropometric datasets. P. L. Q. and M. F. F. C. were responsible for finalising the manuscript. All authors contributed to and approved the final manuscript.

P. D. G., K. M. G. and Y.-S. C. have received reimbursement for speaking at conferences sponsored by companies selling nutritional products. These authors are part of an academic consortium that has received research funding from commercial affiliations such as Abbott Nutrition, Nestec and Danone. None of the other authors report any potential conflict of interest. This does not alter our adherence to British Journal of Nutrition policies on sharing data and materials.

\section{Supplementary material}

To view supplementary material for this article, please visit https://doi.org/10.1017/S0007114519002253

\section{References}

1. Pwint MK, Lee YS, Wong TY, et al. (2013) Prevalence of overweight and obesity in Chinese preschoolers in Singapore. Ann Acad Med 42, 66-72.

2. Chan JC, Malik V, Jia W, et al. (2009) Diabetes in Asia: epidemiology, risk factors, and pathophysiology. JAMA $\mathbf{3 0 1}$, 2129-2140.
3. Simmonds M, Burch J, Llewellyn A, et al. (2015) The use of measures of obesity in childhood for predicting obesity and the development of obesity-related diseases in adulthood: a systematic review and meta-analysis. Health Technol Assess 19, 1-336.

4. Bleich SN \& Vercammen KA (2018) The negative impact of sugar-sweetened beverages on children's health: an update of the literature. BMC Obes 5, 6.

5. Malik VS, Pan A, Willett WC, et al. (2013) Sugar-sweetened beverages and weight gain in children and adults: a systematic review and meta-analysis. Am J Clin Nutr 98, 1084-1102.

6. Savage JS, Fisher JO \& Birch LL (2007) Parental influence on eating behavior: conception to adolescence. J Law Med Ethics 35, 22-34.

7. Park S, Pan L, Sherry B, et al. (2014) The association of sugar-sweetened beverage intake during infancy with sugarsweetened beverage intake at 6 years of age. Pediatrics 134, Suppl. 1, S56-S62.

8. Leermakers ET, Felix JF, Erler NS, et al. (2015) Sugar-containing beverage intake in toddlers and body composition up to age 6 years: the generation R study. Eur J Clin Nutr 69, 314-321.

9. Bleich SN \& Wolfson JA (2015) Trends in SSBs and snack consumption among children by age, body weight, and race/ ethnicity. Obesity 23, 1039-1046.

10. Pan L, Li R, Park S, et al. (2014) A longitudinal analysis of sugarsweetened beverage intake in infancy and obesity at 6 years. Pediatrics 134, Suppl. 1, S29-S35.

11. DeBoer MD, Scharf RJ \& Demmer RT (2013) Sugar-sweetened beverages and weight gain in 2- to 5-year-old children. Pediatrics 132, 413-420.

12. Newby PK, Peterson KE, Berkey CS, et al. (2004) Beverage consumption is not associated with changes in weight and body mass index among low-income preschool children in North Dakota. J Am Diet Assoc 104, 1086-1094.

13. Angkurawaranon C, Jiraporncharoen W, Chenthanakij B, et al. (2014) Urban environments and obesity in southeast Asia: a systematic review, meta-analysis and meta-regression. PLOS ONE 9, e113547.

14. Yu P, Chen Y, Zhao A, et al. (2016) Consumption of sugarsweetened beverages and its association with overweight among young children from China. Public Health Nutr 19 , $2336-2346$

15. Shang XW, Liu AL, Zhang Q, et al. (2012) Report on childhood obesity in China (9): sugar-sweetened beverages consumption and obesity. Biomed Environ Sci 25, 125-132.

16. Ha K, Chung S, Lee HS, et al. (2016) Association of dietary sugars and sugar-sweetened beverage intake with obesity in Korean children and adolescents. Nutrients $\mathbf{8}$, E31.

17. Lin PY, Lin FY, Chen TC, et al. (2016) Relationship between sugar intake and obesity among school-age children in Kaohsiung, Taiwan. J Nutr Sci Vitaminol 62, 310-316.

18. Soh SE, Tint MT, Gluckman PD, et al. (2014) Cohort profile: Growing Up in Singapore Towards healthy Outcomes (GUSTO) birth cohort study. Int J Epidemiol 43, 1401-1409.

19. Lim HX, Toh JY, Tan KH, et al. (2018) Validation of a semiquantitative food frequency questionnaire for 18-month-old toddlers: the GUSTO study. Public Health Nutr 22, 1-11.

20. Sugianto R, Chan MJ, Tai BC, et al. (2019) Relative validity of a quantitative food frequency questionnaire for five-year-old children in an Asian population. J Acad Nutr Diet (In the Press).

21. Goh DY \& Jacob A (2011) Children's consumption of beverages in Singapore: knowledge, attitudes and practice. I Paediatr Child Health 47, 465-472.

22. Aris IM, Bernard JY, Chen LW, et al. (2017) Infant body mass index peak and early childhood cardio-metabolic risk markers in a multi-ethnic Asian birth cohort. Int J Epidemiol 46, $513-525$. 
23. de Onis M, Onyango AW, Borghi E, et al. (2007) Development of a WHO growth reference for school-aged children and adolescents. Bull World Health Organ 85, 660-667.

24. WHO (2018) World Health Organization: Growth reference 5-19 years. http://www.who.int/growthref/who2007_bmi_ for_age/en/ (accessed January 2019).

25. Aris IM, Gandhi M, Cheung YB, et al. (2014) A new populationbased reference for gestational age-specific size-at-birth of Singapore infants. Ann Acad Med 43, 439-447.

26. Pang WW, Aris IM, Fok D, et al. (2016) Determinants of breastfeeding practices and success in a multi-ethnic Asian population. Birth 43, 68-77.

27. WHO (1995) World Health Organization's infant feeding recommendations. Wkly Epidemiol Rec 70, 117-120.

28. Bernard JY, Padmapriya N, Chen B, et al. (2017) Predictors of screen viewing time in young Singaporean children: the GUSTO cohort. Int J Behav Nutr Phys Act 14, 112.

29. Coutinho LM, Scazufca M \& Menezes PR (2008) Methods for estimating prevalence ratios in cross-sectional studies. Rev Saude Publica 42, 992-998.

30. Greenland S (2004) Model-based estimation of relative risks and other epidemiologic measures in studies of common outcomes and in case-control studies. Am J Epidemiol 160, 301-305.

31. Zou GY \& Donner A (2013) Extension of the modified Poisson regression model to prospective studies with correlated binary data. Stat Methods Med Res 22, 661-670.

32. Mickey RM \& Greenland S (1989) The impact of confounder selection criteria on effect estimation. Am J Epidemiol 129, 125-137.

33. Chaidez V, McNiven S, Vosti SA, et al. (2014) Sweetened food purchases and indulgent feeding are associated with increased toddler anthropometry. J Nutr Educ Behav 46, 293-298.

34. Dubois L, Farmer A, Girard M, et al. (2007) Regular sugarsweetened beverage consumption between meals increases risk of overweight among preschool-aged children. J Am Diet Assoc 107, 924-934; discussion 34-35.

35. Ariza AJ, Chen EH, Binns HJ, et al. (2004) Risk factors for overweight in five- to six-year-old Hispanic-American children: a pilot study. J Urban Health 81, 150-161.

36. Yu CJ, Du JC, Chiou HC, et al. (2016) Sugar-sweetened beverage consumption is adversely associated with childhood attention deficit/hyperactivity disorder. Int JEnviron Res Public Health 13, E678.

37. Freedman DS, Wang J, Ogden CL, et al. (2007) The prediction of body fatness by BMI and skinfold thicknesses among children and adolescents. Ann Hum Biol 34, 183-194.

38. Bennette C \& Vickers A (2012) Against quantiles: categorization of continuous variables in epidemiologic research, and its discontents. BMC Med Res Methodol 12, 21.

39. Cantoral A, Tellez-Rojo MM, Ettinger AS, et al. (2016) Early introduction and cumulative consumption of sugar-sweetened beverages during the pre-school period and risk of obesity at 8-14 years of age. Pediatr Obes 11, 68-74.

40. Zheng M, Rangan A, Olsen NJ, et al. (2015) Substituting sugar-sweetened beverages with water or milk is inversely associated with body fatness development from childhood to adolescence. Nutrition 31, 38-44.

41. Ambrosini GL, Oddy WH, Huang RC, et al. (2013) Prospective associations between sugar-sweetened beverage intakes and cardiometabolic risk factors in adolescents. Am J Clin Nutr 98, 327-334.

42. Popkin BM \& Hawkes C (2016) Sweetening of the global diet, particularly beverages: patterns, trends, and policy responses. Lancet Diabetes Endocrinol 4, 174-186.

43. Willson K \& Situ C (2017) Systematic review on effects of diet on gut microbiota in relation to metabolic syndromes. J Clin Nutr Metab 1, 2.

44. Ministry of Education (2018) Getting to know your plate: a guide to healthy eating. http://punggolsec.moe.edu.sg/qql/ slot/u365/CCA/Geting\%20to\%20know\%20your\%20plate\%20$\% 20$ A $\% 20$ guide\%20to\%20healthy\%20eating.pdf (accessed January 2019)

45. Willett W (1998) Nutritional Epidemiology. New York: Oxford University Press.

46. Kipnis V, Subar AF, Midthune D, et al. (2003) Structure of dietary measurement error: results of the OPEN biomarker study. Am J Epidemiol 158, 14-21; discussion 2-6.

47. Szatmari P \& Jones MB (1999) Effects of misclassification on estimates of relative risk in family history studies. Genet Epidemiol 16, 368-381.

48. Kobayashi T, Kamimura M, Imai S, et al. (2011) Reproducibility and validity of the food frequency questionnaire for estimating habitual dietary intake in children and adolescents. Nutr J 10, 27. 